\title{
Is it the Time Now for Cardiac Surgery to Fully Engage in Telemedicine
}

\author{
Omar Lattouf ${ }^{1}$ \\ ${ }^{1}$ Emory University School of Medicine
}

June 28, 2021

\begin{abstract}
Telemedicine, telehealth and artificial intelligence in healthcare are becoming commonly utilized in various medical specialties. The article authored by Dr. Aminah Sallam and colleagues in the Journal provides data in support of the cardiac surgical patients, and the caring cardiac surgeons willingness to adopt telemedicine as a method of connectivity between patient and surgeon.
\end{abstract}

Editorial: Telemedicine in the Era of Coronavirus 19: Implications for Post-Operative Care in Cardiac Surgery

Omar M Lattouf MD PHD FACC FACS

Professor of Surgery, Emeritus

Emory University School of Medicine

Dr. Aminah Sallam and colleagues are to be commended on their article titled "Telemedicine in the Era of Coronavirus 19: Implications for Post-Operative Care in Cardiac Surgery" published in the Journal of Cardiac Surgery.

In this article, the authors reported results of small study on fifty patients who had undergone cardiac operations during the height of the coronavirus pandemic, and underwent follow-up with their surgeons via telephone or via video-supported methods.

Interestingly, the level of satisfaction by both patients and surgeons for utilizing telemedicine was high with, reportedly, all surgeons prefer to see patients post-operatively using telemedicine and $46 \%$ of patients would still take telemedicine post operative visits over in-person visits even if there was no "stay-at-home orders".

These noteworthy findings deserve the attention of every practicing cardiac surgeon in this ever-changing environment, where external pressures on the cardiac surgical practice are mounting. One such example of important and relevant external pressures is the impending threat for upward of $20 \%$ of Medicare reimbursement cuts for cardiothoracic surgery remains real and would have catastrophic impact on the practice of cardiothoracic surgery. Thus it behooves us to deploy every possible method to enhance our services to our patients, protect the cardiac surgical specialty and our ability to provide life-saving care to our patients. ${ }^{1}$

In this era were simulation-based training has become common in cardiac surgery ${ }^{2}$ and virtual reality has become an important aspect of our everyday life ${ }^{3}$, perhaps it is time for us, cardiac surgeons to "jump on the band wagon" and not let this opportunity to expand our connectivity, and bonding, with our patients and their families slip by us. 
Cardiac surgeons, as healthcare leaders who have advanced the care of the cardiac patients and have been critical in introducing cutting edge technologies into the cardiac care in particular ${ }^{4}$ and into the entire field of medicine in general, should continuously examine ways to improve patient care irrespective of patients' geographic locations. And as we have always done, we should aspire to improve the quality of patient care in every way possible ${ }^{5}$, inside the walls of our hospitals and clinics as well as when patients are in their own home environment.

The use of telemedicine is one such way through which we can provide virtual care, health education and expanded connectivity.

One of the many important lessons we have learned since the Covid pandemic is that we have to be innovative and creative in order to provide critical patient care and help patients stay healthy ${ }^{6}$.

Telemedicine has been shown over the last several decades to bring much needed health services to patients in remote areas ${ }^{7}$. NASA's unique contribution to telemedicine by creating technologies to provide medical care to astronauts during space missions have benefitted not only the astronauts, or the American citizens but has also served people from all over the world ${ }^{8}$.

Cardiac diseases afflicts millions of people around the globe, is one of the leading causes of death and is associated with multiple comorbid factors such as stroke, depression, obesity, hypertension, diabetes and smoking ${ }^{9}$.

Rural America has its not-small share of patients who are suffering from CV diseases, many at remote areas with lesser access to cardiovascular specialists than urban populations ${ }^{10}$.

Patients who are in need of pre-operative cardiac care as well as patients who are in need of post-operative follow-up would benefit from telemedicine-supported visits. Such patients could remain in frequent contact via telemedicine with their providers until their conditions stabilize, wounds heal and are back into being independent and capable of self-care.

In conclusion, tele-medicine in cardiac surgery has the potential of many benefits such as:

1. Allow early introductions of patients to surgeon while patient is still at home or at a remote clinical facility.

2. Video-personalized meeting in advance of patient scheduled admission to hospital for planned surgery with patient and their families or care-providers without burdening the entire family to travel and incur the cost and lengthy times of travel and waiting. Family members from different locations could attend such video-supported session, thus enabling multiple parties to join, meet the surgical team and learn about the details of the operative procedure, associated benefits and risks and have opportunity to ask questions. .

3. More frequent video-supported visits of patient post discharge while at home.

The time is now for cardiac surgeons to embrace tele-medicine and incorporate it into our every-day practice.

References

1. Alan M. Speir, Courtney Yohe, Stephen J. Lahey, Julie R. Painter, and Francis C. Nichols, on behalf of the STS Workforce on Health Policy, Advocacy, and Reform, and the Workforce on Coding and Reimbursement. 2020 Medicare Final Payment Rule: Implications for Cardiothoracic Surgery. Ann Thorac Surg 2020; 109:313-6.

2. Igo B. Ribeiro, Janet M. C. Ngu, Buu-Khanh Lam, and Roger A. Edwards. Simulation-Based Skill Training for Trainees in Cardiac Surgery: A Systematic Review. Ann Thorac Surg 2018; 105:972-82.

3. KIMBERLY J. MILLER, BROOKE S. ADAIR, ALAN J. PEARCE, CATHERINE M. SAID, ELIZABETH OZANNE, MEG M. MORRIS. Effectiveness and feasibility of virtual reality and gaming system use at home by older adults for enabling physical activity to improve health related domains: a systematic review. Age and Ageing 2014; 43: 188-195

4. Richard J. Shemin. The Future of Cardiovascular Surgery.Circulation . 2016; 133:2712-2715 
5. Lawrence H. Cohn. WHAT THE CARDIOTHORACIC SURGEON OF THE TWENTY-FIRST CENTURY OUGHT TO BE. J Thorac Cardiovasc Surg 1999; 118:581-7.

6. Judd E. Hollander, and Brendan G. Carr. Virtually Perfect? Telemedicine for Covid-19. NEJM 382;18 April 30, 2020; 1679-1681.

7. Michael E. DeBakey. Telemedicine Has Now Come of Age. Telemedicine Journal. Jan 1995.3-4. Volume: 1 Issue 1: January 29, 2009.

8. Doarn CR, Nicogossian AE, Merrell RC. Application of telemedicine in the United States Space Program. Telemed J. 1998; 4(1):19-30.

9. Heval M Kelli, Ibrahim Kassas and Omar M Lattouf. Cardio Metabolic Syndrome: A Global Epidemic. J Diabetes Metab 2015, 6:3

10. Julie A. Nelson, and Barbara Stover Gingerich. Rural Health: Access to Care and Services. Home Health Care Management \& Practice 22(5) 339-343 